\title{
Soziologische Fachtexte aus Korpusperspektive. Zum Auffinden indirekter Rede in den Annali di Sociologia/Jahrbüchern für Soziologie
}

\author{
Antonie Hornung (Zürich/Modena)
}

\begin{abstract}
This paper deals with the question of which search strategies are adapted to corpus analysis when trying to point out textual phenomena such as, for instance, indirect and direct speech. My study investigates the quantity and the various forms of direct and indirect speech used to make references to others' research. It draws upon the corpus of the German originals published in the Annali di Sociologia from 1991 to 2002, a journal edited in German and Italian (originals and translations in both languages) by the University of Trento, Italy.
\end{abstract}

Im Gedenken an John Dewey

\section{$1 \quad$ Suchgegenstand indirekte Rede}

Der vorliegende Beitrag beschäftigt sich mit der Frage, welche Suchstrategien das Auffinden kontextrelevanter Textphänomene in Korpora erleichtern können. Im Rahmen vergleichender Untersuchungen zur deutschen und zur italienischen Wissenschaftssprache, die vom interuniversitären Forschungsteam Modena - Bergamo - Firenze ${ }^{1}$ im Rahmen eines nationalen Forschungsprojektes (PRIN 2005) durchgeführt wurden und werden, geht es neben den spezifischen Elementen der Mikroebene auch um die Charakteristika der textuellen Mesoebene und der gesamten Textstruktur. Während sich aber die Analyse einzelner sprachlicher Mittel mit Hilfe der vorhandenen technischen Ausstattung relativ einfach bewerkstelligen lässt, führen Abfragen, die Einblicke in komplexere Textbausteine gewähren und die Analyse der Wege der Gedankenabwicklung ermöglichen sollen, immer wieder in Sackgassen.

Der Textbaustein, an dem die Problematik der Suche im Folgenden expliziert werden soll, ist die indirekte Rede. Indirekte Rede ist die Wiedergabe "propositionalen Wissens, das der Sprecher selbst oder ein anderer Sprecher, und zwar zu einem anderen Sprechzeitpunkt als dem gerade aktualen Sprechzeitpunkt oder in einer nur gedachten Äußerungssituation" (Zifonun/Hoffmann/Strecker 1997: 1753) zum Ausdruck gebracht hat. In wissenschaftlichen Texten dient sie - ähnlich der direkten Rede - der Sichtbarmachung dieses Wissens in neuem Kontext. Damit stellt sie es einem neuen Empfängerkreis zur Verfügung, macht Positionen deutlich, bereichert den Argumentationszusammenhang, fungiert u. U. als Autoritätsbeweis und markiert letztlich auch die Seilschaftszugehörigkeit einer Verfasserin oder eines Verfassers. (vgl. Jakobs 1994; Panther 1981; Adamzik 1998). Kurz, indirekte Rede kann als

\footnotetext{
1 Das Team Modena - Bergamo - Firenze hat sich im Rahmen eines nationalen Forschungsprojekts zum Thema "Lingue di cultura in pericolo? Il caso del tedesco e dell'italiano nella formazione accademica" konstituiert und konzentriert seine vergleichenden Untersuchungen auf die Herausarbeitung textueller Merkmale der deutschen und der italienischen Wissenschaftssprache (cf. Heller 2008a).
} 
ein Element der Vielstimmigkeit in Texten (vgl. dazu Bachtin 1979; Pérennec 2000/2003) auf wichtige inner- und außertextuelle Vernetzungen verweisen.

\section{Möglichkeiten und Grenzen von Sketch engine}

Das für die im Rahmen der Weiterentwicklung des oben erwähnten Forschungsprojekts geplanten vergleichenden wissenschaftssprachlichen Untersuchungen zur Verfügung stehende Korpus umfasst die ab 1991 erschienenen Bände der Annali di Sociologia/Soziologisches Jahrbuch, einer wissenschaftlichen Zeitschrift, die von der Soziologischen Fakultät der Universität Trento zweisprachig herausgegeben wird. Es handelt sich dabei, was den Sprachgebrauch angeht, um ein Kreuzkorpus, d.h. die je Band etwa zur Hälfte italienisch- und deutschsprachigen Beiträge stehen jeweils auch übersetzt zur Verfügung. Das Korpus eignet sich deshalb sehr gut für deutsch-italienisch ausgerichtete komparatistische Analysen. Im Rahmen der Arbeiten für das Südtiroler Korpus wurden die Bände 7-16 (1991 bis einschließlich 2002; insgesamt 11 Bde. bzw. Teilbände) korpuslinguistisch aufgearbeitet; sie stehen dem interuniversitären Forschungsteam Modena - Bergamo - Firenze über die leicht handhabbare Suchmaske der Sketch engine zur Verfügung (vgl. Abb. 1; zur genaueren Beschreibung des Korpus vgl. Heller 2008: 106f.).

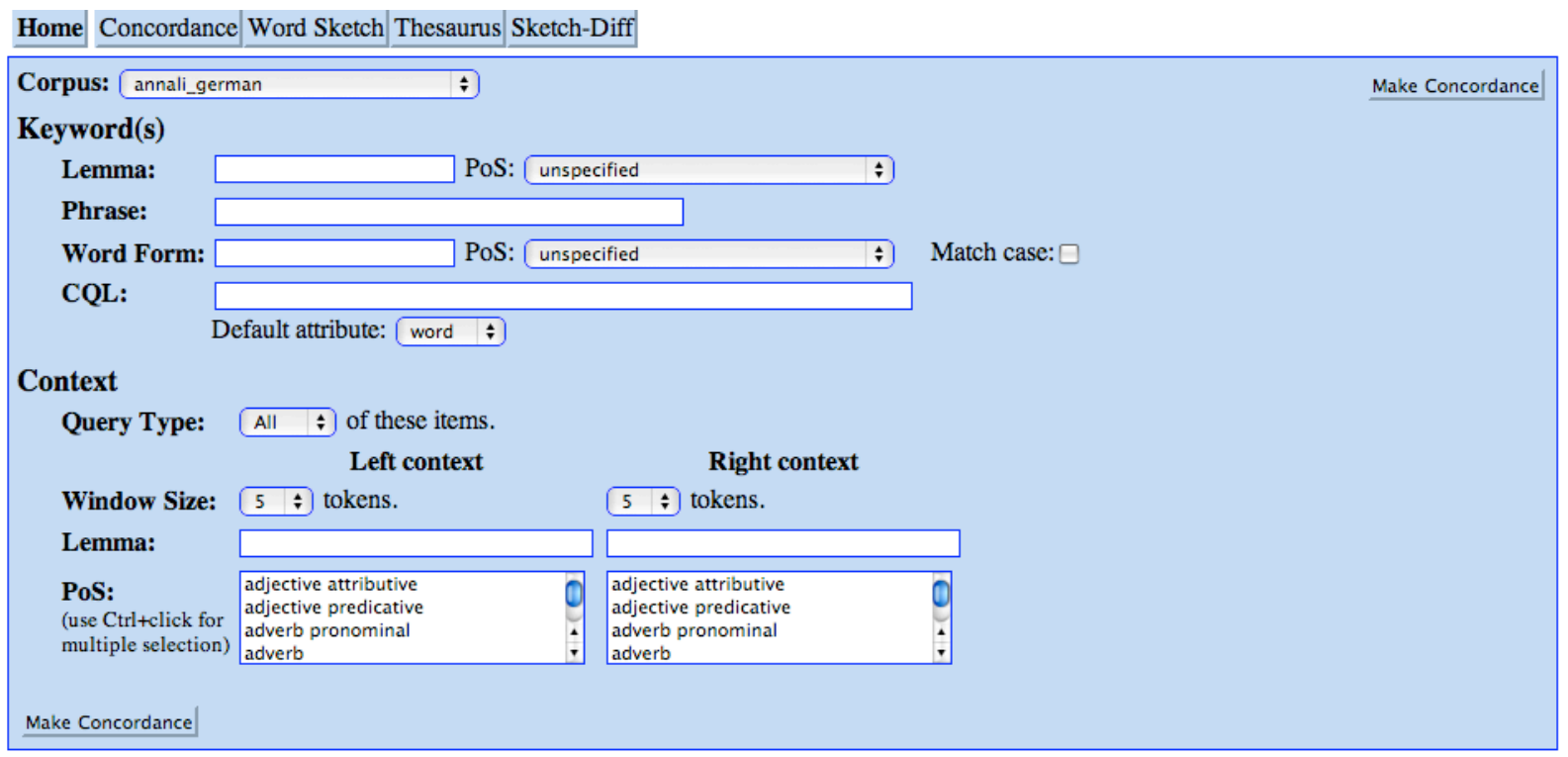

Abb. 1: Suchmaske für die Annali di Sociologia

\subsection{Einfache Suchfiguren}

Zugriffsweisen auf das Korpus, an die man bezüglich der Suche nach indirekter Rede vermutlich zuerst denkt, bestehen in der Abfrage von Verba dicendi bzw. scrivendi oder auch von Verbformen im Konjunktiv I oder II und, last but not least, in der Suche nach Namen von Experten, über deren Nennung man, so wäre die Hypothese, relativ schnell zu den gesuchten Stellen vorstoßen müsste. Allerdings erlauben beide ersteren Zugriffsweisen nur bedingt ein systematisches Vorgehen, weil das einschlägige Verbenarsenal schlicht zu umfangreich ist. Ein weiteres Hindernis besteht darin, dass die Eigennamen im vorliegenden Korpus (noch) nicht getaggt sind, d.h. sie sind nur über die Funktion Phrase auffindbar, was die Suchkombinatorik erheblich beschränkt (hierzu weiter unten in diesem Beitrag). Probeläufe mit Verben wie sagen, bemerken, erwähnen, schreiben, sich beziehen auf, widersprechen, kritisieren, einige davon auch in Kombinationen mit so oder wie sind wenig ergiebig. Was sich hingegen als überschaubares und annähernd systematisches Vorgehen anbietet, ist die 
Suche nach den Konjunktiv-Formen der Hilfsverben und der Modalverben. Dieser Weg soll im Folgenden auf seine Brauchbarkeit überprüft werden.

\subsubsection{Suchfigur 1: Konjunktivformen der Hilfsverben "haben", "sein" und "werden"}

Gemäß den geltenden Regeln bezüglich des Gebrauchs der indirekten Rede ist davon auszugehen, dass das Verb in ihr im Konjunktiv I bzw. - im Fall gleichlautender Formen von Indikativ und Konjunktiv I - im Konjunktiv II zu stehen hat. Da anzunehmen ist, dass Hilfsverben auch in wissenschaftlichen Texten häufig verwendet werden, müsste die Suche mit den einschlägigen Konjunktivformen eigentlich erfolgreich sein.

\subsubsection{Grammatikalisch eindeutige Formen vs. versuchte Vollständigkeit: Bsp. "habe"}

Die Entwicklungsgeschichte der deutschen Verben hat dazu geführt, dass wir heute eine Fülle von homonymen Formen haben, deren grammatikalische Zuordnung ohne einschlägigen Kontext nicht eindeutig sein kann. Im Folgenden werden deshalb die Ergebnisse einer Suchabfrage, die sämtliche Belege für die 3. Pers. Sg. von "haben" ergibt, den Resultaten von Suchabfragen mit grammatikalisch eindeutigen Formen gegenübergestellt.

Die Suche nach der Form "habe" ergibt insgesamt 219 Belege (vgl. a), davon entstammen 104 einer indirekten Rede, also knapp die Hälfte. In neun Fällen häufen sich die Belege aus dem gleichen Text (drei oder mehr, bis zu sieben Konjunktivformen pro Textstelle), was auf umfangreichere indirekt referierte Aussagen hindeutet.

a) Phrase: "habe ":

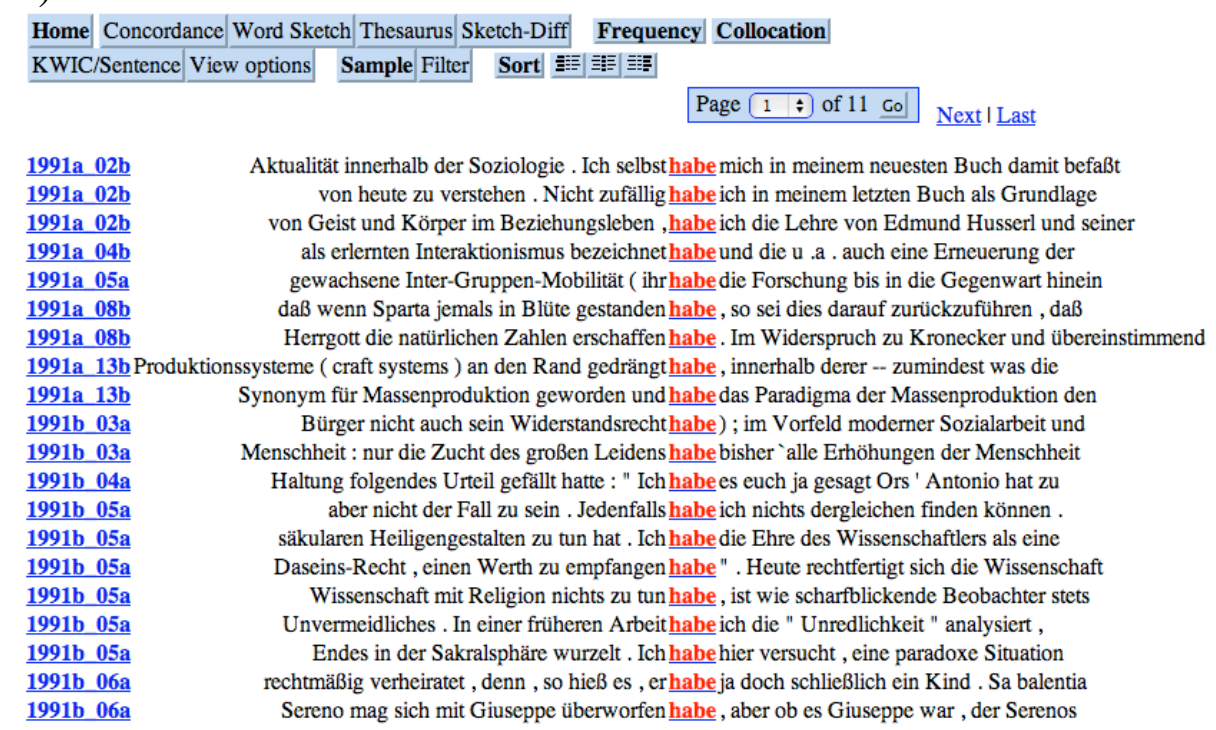

Das Gesamtergebnis lässt sich durch ein Diagramm übersichtlich darstellen (vgl. Diagramm 1). In 48 verschiedenen Texten der Annali konnten mit dem Suchwort "habe" Belege für indirekte Rede festgestellt werden; in 28 davon finden sich jeweils zwei oder mehr Belege. Die je zwei Annali-Bände der Jahre 1992 und 1993 scheinen besonders reich an Belegen zu sein, ebenso der Band von 1996 und der von 2002. Man kann auch erkennen, dass sich in den Originalen (Sigle ...a, Bsp. 1991a 05a) mehr Belege finden als in den Übersetzungen (Sigle ...b, Bsp. 1991a 13b). 


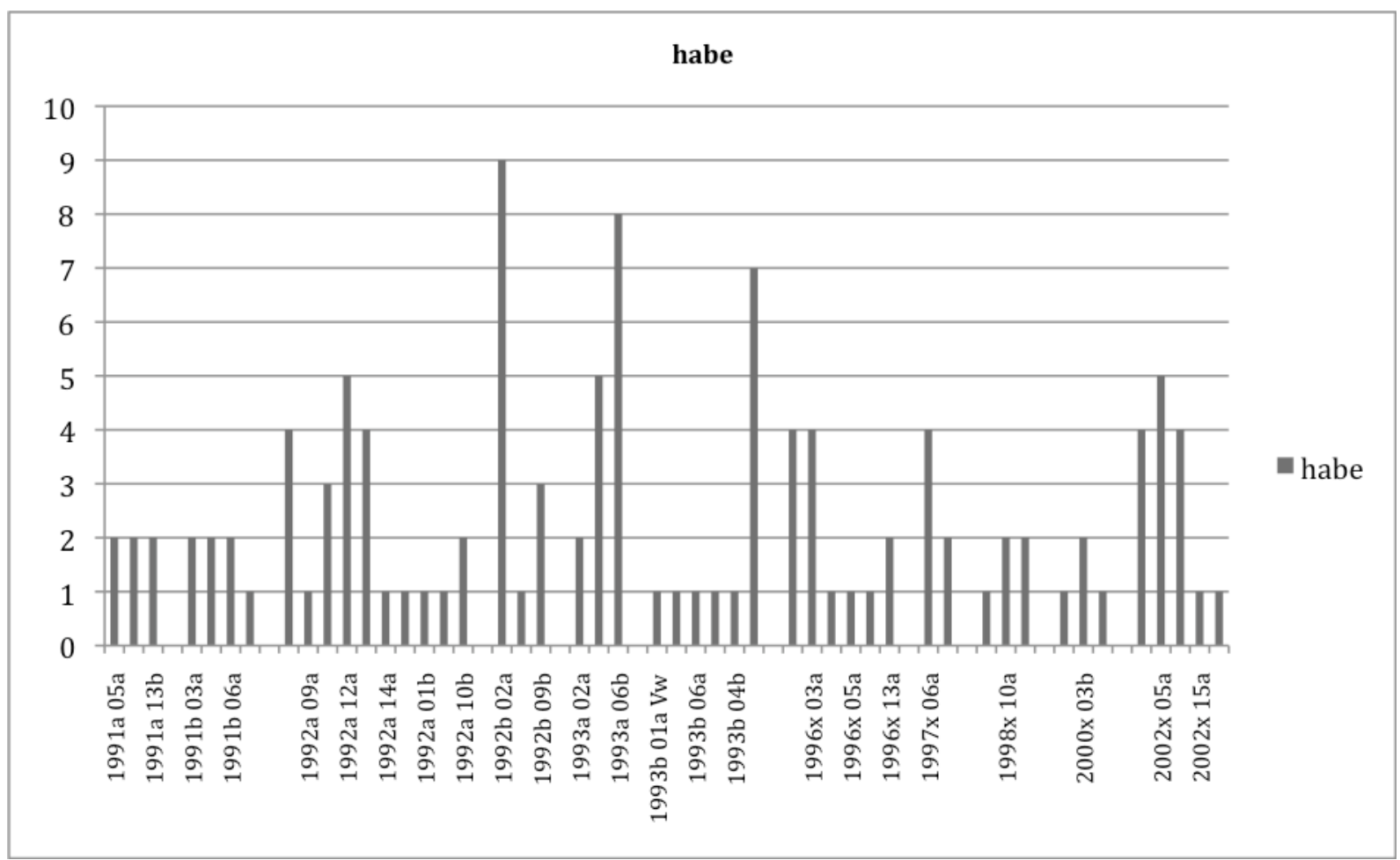

Diagramm 1: Belegstellen für "habe"

Angesichts der aufwendigen Prozedur des Handverlesens der mehrdeutigen Form "habe" stellt sich die Frage, ob sich die Suche nicht durch kombinatorische Anfragen beschleunigen lässt. Beispielsweise ergab sich aus der Beleganalyse bei Suche a) vierzehn Mal in unmittelbarer Nachbarschaft zum Suchwort der Name eines Soziologen und fünfmal der Bezug zur Disziplin (vgl. b). Eine kleine Kontrollsuche nach "habe" plus "Soziologie" im rechten Kontext bestätigt diese Ergebnisse:

b) Phrase: "habe" plus im rechten Kontext (7 Token): "Soziologie"

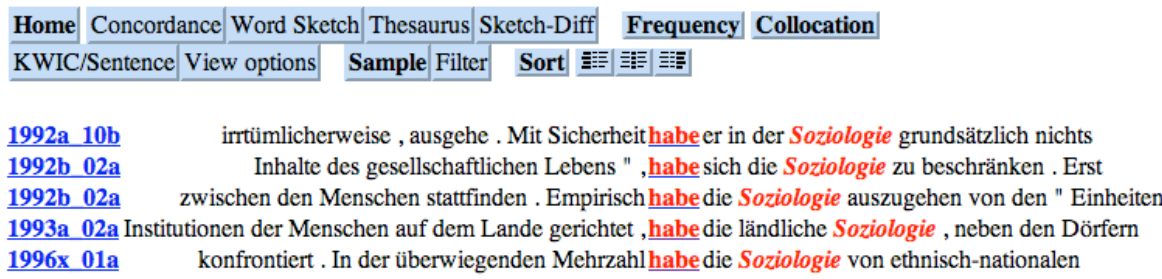

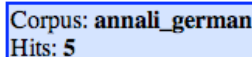

conc description

Eine höhere Anzahl Belege bei größerer Suchsicherheit versprechen allerdings Formen wie "er/sie/es habe" bzw. in der invertierten Form "habe er/sie"2. Sie sind eindeutige Konjunktiv-I-Formen und dürften mit größter Wahrscheinlichkeit auf indirekte Reden zuführen.

2 Bei der Formulierung "habe es" kann es sich auch um den Indikativ der ersten Person Präsens ("Ich habe es") handeln, deshalb wird diese Wortkombination hier vorderhand beiseite gelassen. 
c) Phrase: "er habe":

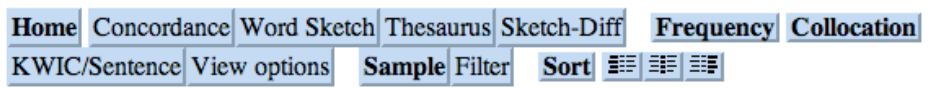

1991b 06a rechtmäßig verheiratet, denn, so hieß es, er habe ja doch schließlich ein Kind . Sa balentia

1992a 04a seinem 70 . Geburtstag bemerkte Dilthey, er habe versucht, im Sinne der universalhistorischen 1992a 10b Gemeinschaftswerk vorschlägt, antwortet ihm Simmel, er habe seit Jahren jedes praktische Interesse

1993b 04b Educatore " veröffentlichten Aufsatz berichtet, er habe in Basel einen "wunderschönen Film gesehen

1998x 10a Gauner keine Vorwürfe , als er mir erzählte, er habe eine Brieftasche " gefunden , bevor sie

$\underline{\text { 2002x 08a }}$ Ezechiel sei nicht " normal " gewesen bzw .er habedie " Normalität " im damaligen Israel

Das Ergebnis ist durchaus zufriedenstellend: Zwei der sechs Belege bringen in unmittelbarer Nachbarschaft der gesuchten Verbform den Autorbezug und mit den Namen Dilthey bzw. Simmel den Hinweis, dass hier Klassiker der Soziologie zitiert werden. Beleg vier macht zudem die Quelle der indirekten Aussage inkl. bibliographischer Angaben bei erweiterter Kontextsuche bekannt, während die anderen Belege für indirekte Rede auf allgemeine Erzählkontexte verweisen, deren genaue Herkunft jedoch auch bei links- und rechtserweiterter Kontextsuche im Vagen bleibt.

d) Phrase: "habe er":

\section{Home Concordance Word Sketch Thesaurus Sketch-Diff Frequency Collocation KWIC/Sentence View options Sample Filter Sort 瑟琵瑟}

1991b 06a sprechen " ( s'omine bonu narrat in cara ), habe er hinterrücks gehandelt, hieß es . Immer $\underline{1992 \mathrm{a} 10 \mathrm{~b}}$ irrtümlicherweise, ausgehe . Mit Sicherheit $\underline{\underline{\text { habe er }}}$ in der Soziologie grundsätzlich nichts

e) Phrase: "sie habe":

\section{Home Concordance Word Sketch Thesaurus Sketch-Diff Frequency Collocation KWIC/Sentence View options Sample Filter Sort 瑟琵严}

1991b 10b Frau in der allgemeinen Meinung bestand, sie habe sich keinem anderen Mann hingegeben als 1996x 04a eine Geschichte propagiert, glaubt man, sie habe eine . Man glaubt, daß die Homogenität

\section{f) Phrase: "habe sie":}

\section{Home Concordance Word Sketch Thesaurus Sketch-Diff Frequency Collocation KWIC/Sentence View options Sample Filter Sort 琵瑟瑟}

1996x 01a Beschäftigung mit solchen Phänomenen aufdrängte , habe sie sich dieses unliebsamen Gegenstandes weitgehend

\section{g) Phrase: "es habe":}



1997x 06a vorausgesetzt , aber nicht eingeräumt, es habe einen -- , ohne politische ' Maschine

Corpus: annali german

Mit Ausnahme des unter d) aufgeführten zweiten Beispiels (1992a 10b) und des Beispiels e) stellen alle hier zitierten Ausschnitte indirekte Wiedergaben nicht $\mathrm{zu}$ identifizierender Erstverfasser dar, während sich bei genauerer Untersuchung sowohl der Beleg 1992a 10b wie auch 1996x 01a in Hinblick auf die Frage der Polyphonie in soziologischen Fachtexten als Trouvaillen entpuppen. Der erstere gehört nämlich zu der unter c) belegten Stelle aus dem gleichen Text und ist Teil einer mehrere Sätze umfassenden, ausgiebigen Passage, die Simmels Begründung seiner Abkehr von der Soziologie in indirekter Rede ausführlich wiedergibt. Und auch der zweite Beleg verweist auf eine Textpassage, innerhalb der der Gebrauch der indirekten Rede der Verteidigung der Disziplin und der Abgrenzung gegenüber ihren Nachbardisziplinen dient. 


\subsubsection{Indirekte Rede vs. Formulierungsmuster: Bsp. "sei"}

Die grammatikalisch eindeutige Form "sei" bzw. "seien" scheint ein idealer Marker für indirekte Rede zu sein. Allerdings schenkt bereits das erste Blatt neben den zahlreichen Belegstellen für indirekte Reden den Schlüssel zu einem anderen textuell interessanten sprachlichen Mittel, nämlich textkommentierenden Aussagen (vgl. zu den lernpositiven Zufällen der Arbeit mit Korpora Zanin 2007a und 2007b). Vier Belege finden sich allein in dem Aufsatz von 1991a 04b: "Einstweilen sei"; "An dieser Stelle sei"; "Dennoch sei"; "Dazu sei jedoch". Diese katadeiktischen Formulierungsmuster entstammen einer Übersetzung aus dem Italienischen, ein eher überraschender Befund (vgl. Heller 2008b: 130-132), dem andernorts nachzugehen sein wird.

h) Phrase: "sei":



Die Analyse der 472 Belege bringt weitere Formulierungsmuster zutage, die idealerweise auszuschließen wären. Es sind die einen Nebensatz einleitenden Einschränkungen des Typs "es sei denn, dass" oder "sei es, dass". Mit "sei" als Phrase in der Suchmaske, Query Type auf none gestellt, linker Kontext mit Token 1 "es", rechter Kontext ebenfalls mit Token 1 "denn" (vgl. i) wird im linken Kontext "es" und im rechten "denn" ausgeschaltet. 
Home Concordance Word Sketch Thesaurus Sketch-Diff

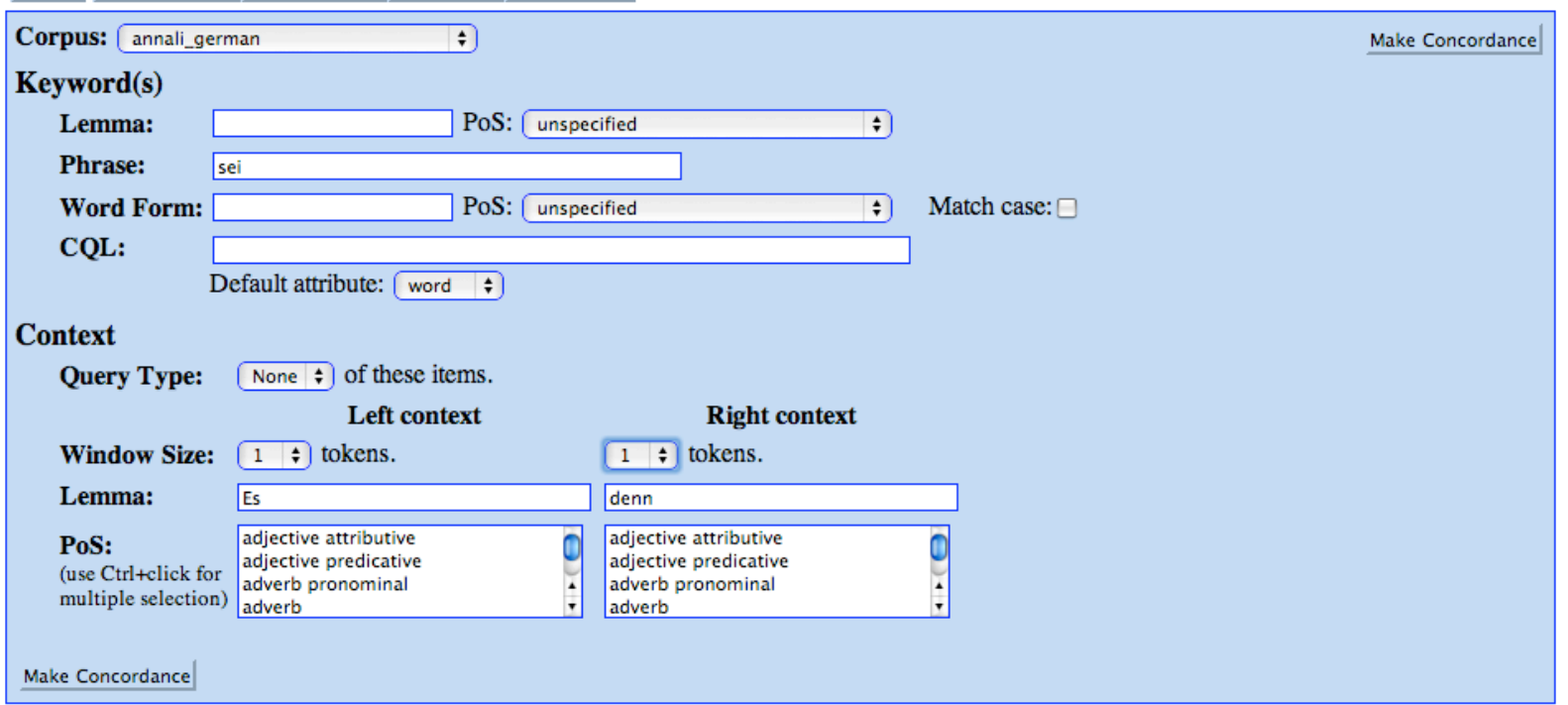

Abb. 2: Ausschluss von "es sei denn"

Es verbleiben 461 Belege von 472 (Belege für "sei"). Ein Kontrollversuch, bei dem die Belegstellen für "es sei denn" abgefragt werden, ergibt 11 Belege und bestätigt die Zuverlässigkeit des gewählten Ausschlussverfahrens.

i) Phrase: "es sei denn":

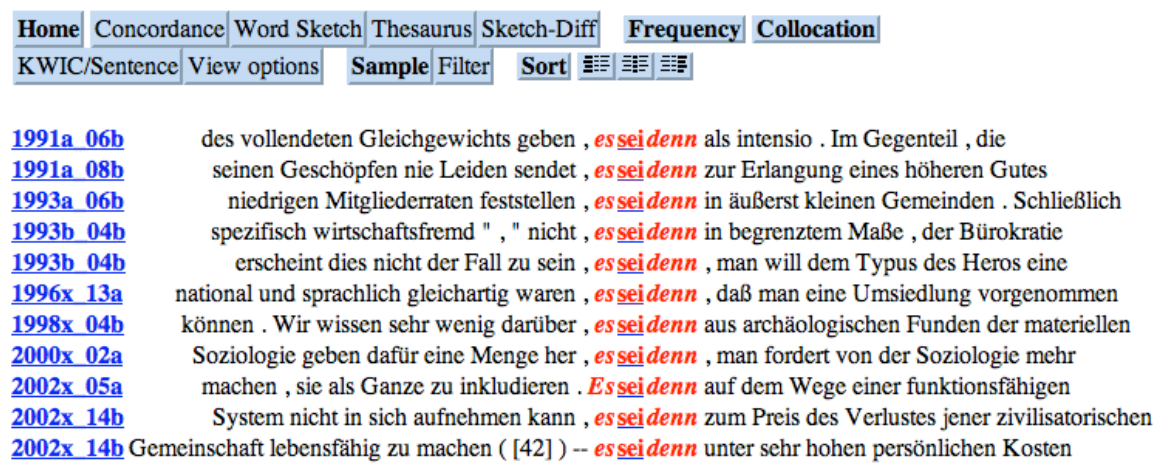

An diesem Punkt zeigen sich allerdings die Grenzen der verfügbaren Suchmaschine. Sketch engine erlaubt nur ein Ausschlussverfahren (vgl. Bsp. h), jedoch nicht die Addition des Ausschlusses verschiedener Formulierungsmuster bei gleicher Suchanfrage. Eine manuelle Durchsortierung der verbliebenen 461 Belege für "sei" ist also unumgänglich. Sie fördert 296 Belege für indirekte Reden zutage. Dabei sind starke Häufungen des Hilfsverbgebrauchs festzustellen; in einem einzigen Text finden sich insgesamt 18 Belege, einmal zwölf, zweimal elf, einmal zehn, zweimal acht, dreimal sieben, fünfmal sechs, fünfmal fünf, neunmal vier, dreizehn Mal drei und fünfzehn Mal Doppelbelege. Solche Häufungen deuten auf umfangreichere Stellen hin.

Auch die Suche nach der Pluralform "seien" beschert reiche Ernte. 112 der 125 Belege für "seien" stellen sich als indirekte Reden heraus; elfmal finden sich drei und mehr Belege im gleichen Text, einmal sogar sechs. Sehr häufig sind Doppelbelege, sie finden sich in insgesamt 19 verschiedenen Texten. In 51 Fällen sind in den gleichen Texten auch Singularbelege zu verzeichnen, vermutlich alles vielversprechende Stellen, die für die weitere Untersuchung der textuellen Funktion der indirekten Rede von Bedeutung zu sein scheinen.

Die Suche mit "sei" hat sich, obwohl hier wesentlich mehr Beispiele auszuschließen waren als bei der Suche mit "habe", als ergiebiger erwiesen (vgl. Diagramm 2). In insgesamt 96 von 143 
Beiträgen aus den Annali konnten Belege für indirekte Rede gefunden werden. Darunter befinden sich immerhin 12 Texte mit zehn und mehr Einzelbelegen. Auch für "sei/seien" trifft man mehr Belege in den je zwei Teilbänden von 1991 und 1992 sowie im Band von 1996 und 2002 an; indirekte Reden finden sich aber auch nicht selten in den beiden Teilbänden von 1993 wie auch im jeweils einzigen Band der Jahre 1997, 1998 und 2002. Nimmt man die Belegstellen für "habe" und "sei/seien" zusammen, so sind vorläufig nur mehr 30 der 143 in den Annali publizierten Aufsätze auszuschließen. Es gibt keinen Band, in dem nicht in mindestens zwei Dritteln der Beiträge eine indirekte Rede vorkommt. Damit zeigt sich bereits an dieser Stelle, dass indirekte Rede in den vorliegenden sozialwissenschaftlichen Texten ein nicht unwichtiges Formulierungskonzept darzustellen scheint.

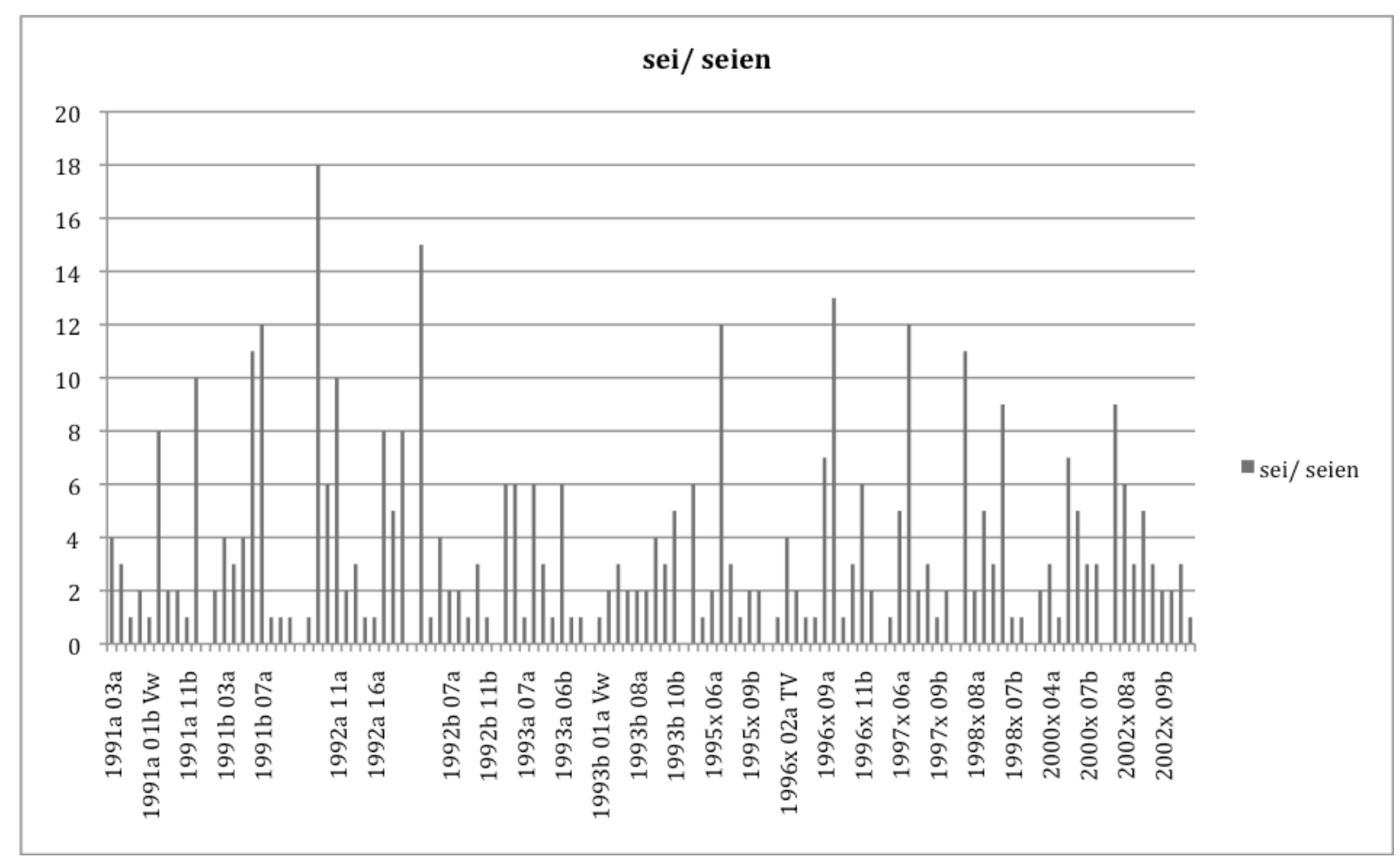

Diagramm 2: Belegstellen für "sei"/"seien"

\subsubsection{Futur oder Passiv: Bsp. "werde"}

Um die Suche nach indirekter Rede via Hilfsverben zu komplettieren, und da sich indirekte Reden möglicherweise auch auf zukunftsgerichtete Aussagen beziehen, ist auch die 3. Pers. Sg. von "werden" einer korpuslinguistischen Abfrage zu unterziehen.

j) Phrase: "werde"

Schließt man die Form des Futur Indikativ der 1. Pers. Sg., die in wissenschaftlichen Texten häufig im Zusammenhang katadeiktischer textkommentierender Formeln anzutreffen ist, aus (vgl. Abb.3), so erhält man mit großer Wahrscheinlichkeit die gleiche Form als Passivmarker, und Passiv ist in fachwissenschaftlichen Texten als eine der Möglichkeiten sachlicher Fomulierung häufig zu erwarten. Von den insgesamt 101 Belegen für "werde" bleiben nach dem Ausschlussverfahren 51, von denen nach genauerer Untersuchung 43 als Hinweise auf indirekte Reden identifiziert werden können. 
Home Concordance Word Sketch Thesaurus Sketch-Diff



Abb. 3: Ausschluss von: "ich werde/werde ich"

Die Suchaktion mit den Konjunktiv-I-Formen der 3. Pers. der Hilfsverben "haben", "sein" und "werden" hat, so kann zusammenfassend gesagt werden, eine stattliche Anzahl von Belegen für indirekte Rede ergeben. Da die jeweiligen Ergebnisse konsequent in eine Excel-Tabelle übertragen wurden, können Überblicksdiagramme erstellt werden, die ihrerseits die Zusammenschau der verschiedenen Belegtypen je Aufsatz ermöglichen. Damit ist ein Weg aufgezeigt, der zu denjenigen Texten aus den Annali führt, in denen vermehrt indirekte Rede gebraucht wird (vgl. exemplarisch für Teilband 1991a Diagramm 3).



Diagramm 3: Daten für 1991a 


\subsubsection{Suchfigur 2: Modalverben im Konjunktiv I Singular}

Die Konjunktivformen der ersten und der dritten Person Singular der Modalverben "müssen", "können", "dürfen", "sollen" und "wollen" sind im Unterschied zu einigen der oben untersuchten Formen eindeutig Konjunktiv. Sie führen mit großer Wahrscheinlichkeit auf Kontexte $\mathrm{zu}$, in denen indirekte Rede im Spiel ist. Deshalb ist davon auszugehen, dass die Belege für sie die Ergebnisse der ersten Suchrunde ergänzen können.

a) Phrase: "müsse":

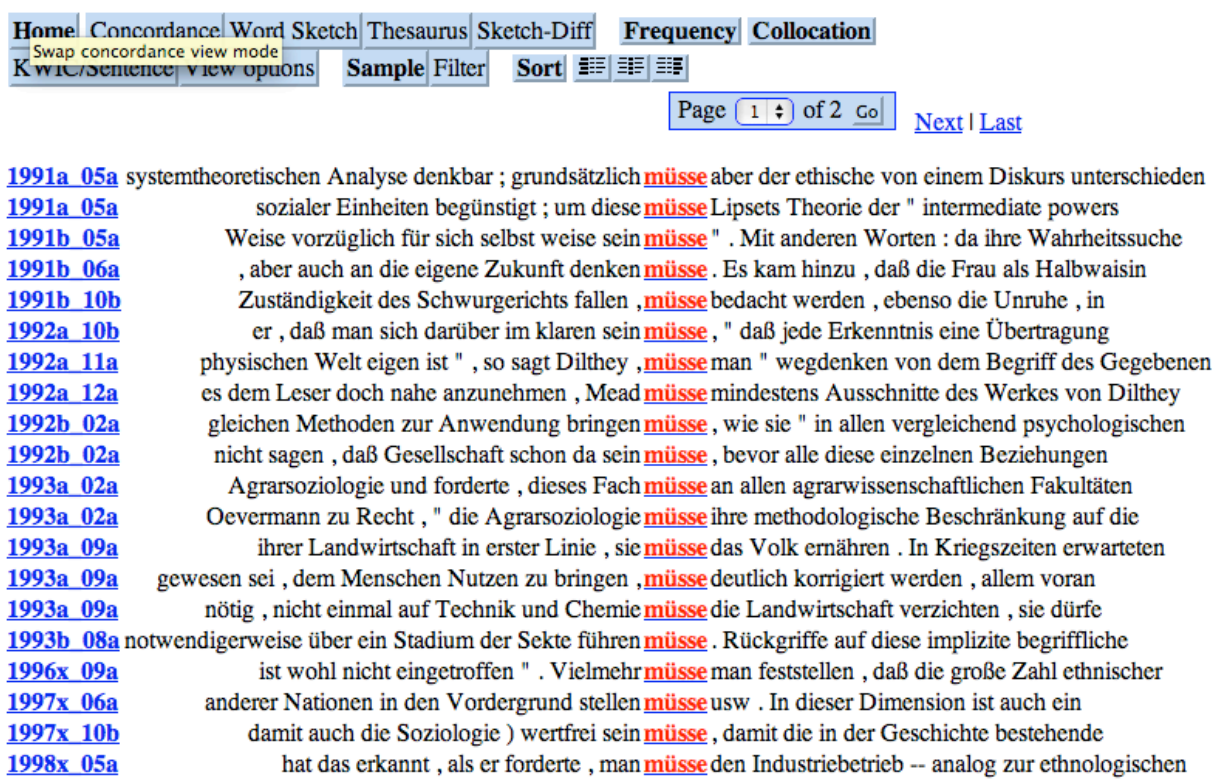

In der Tat sind 27 von 28 Belegen für "müsse" Bestandteil irgendeiner indirekten Rede. Und sämtliche Belege für "könne" (68) und "dürfe" (10) sowie acht von neun Belegen für "solle" und sieben von neun für "wolle" führen zum Gesuchten. Kombiniert man diese Belege mit denen für die Hilfsverben (vgl. Diagramm 4), so scheint sich die Annahme zu bestätigen, dass beim Gebrauch der indirekten Rede jeweils verschiedene sprachliche Mittel variiert werden, was für die korpuslinguistische Suche insofern ein Gewinn ist, als Fälle größerer Ballung von Belegen pro Text als Hinweis auf die Wichtigkeit der Belegstelle verstanden werden dürfen. 


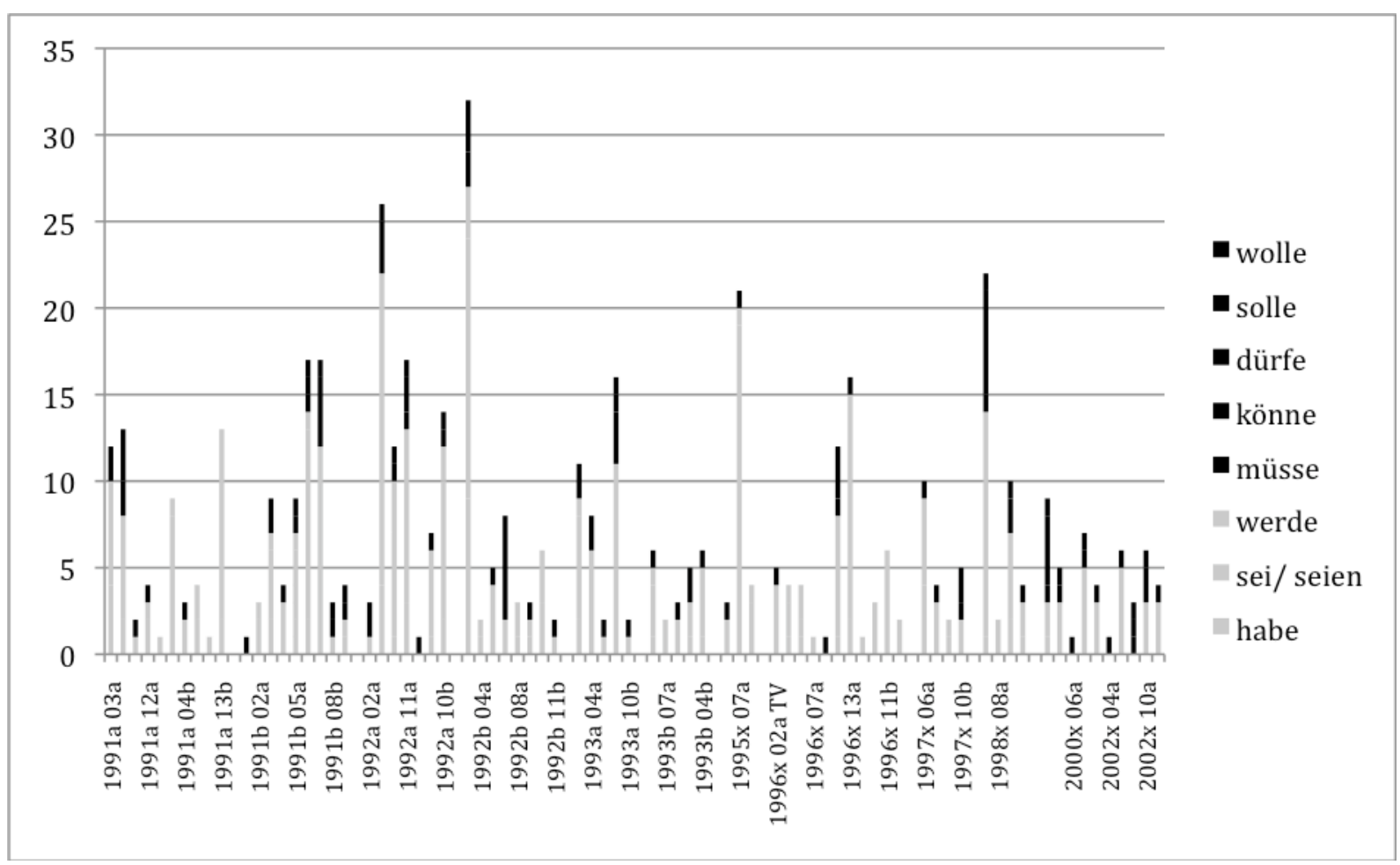

Diagramm 4: Belege für Hilfsverben (hellgrau) und Modalverben (schwarz) im Überblick

Auffallende Unterschiede zwischen den deutschsprachigen Originalen und den Übersetzungen sind anhand der bisher gewonnenen Ergebnisse nicht zu erkennen. Es kann lediglich höchst allgemein festgestellt werden, dass in den aus dem Italienischen ins Deutsche übersetzten Annali-Beiträgen tendenziell weniger Belege für die entsprechenden Formen zu finden sind als in den auf Deutsch verfassten (vgl. Tab. 1). Dies deutet allenfalls darauf hin, dass in den italienischsprachigen Originalen weniger häufig indirekte Rede verwendet wird als in den deutschsprachigen. $\mathrm{Ob}$ dieser Befund möglicherweise mit unterschiedlichen disziplinspezifischen Gepflogenheiten bei der Redewiedergabe zu tun hat, ob er einer grundsätzlich anderen Art der Darstellung zu verdanken ist, oder ob er lediglich durch unterschiedliche Textlängen beeinflusst ist, kann jedoch nur anhand der italienischen Originale genauer untersucht werden.

\begin{tabular}{|l|l|l|l|l|l|l|l|l|}
\hline & habe & $\begin{array}{l}\text { sei/ } \\
\text { seien }\end{array}$ & werde & müsse & könne & dürfe & solle & wolle \\
\hline $\begin{array}{l}\text { Originale } \\
\text { (insges. 75 Beiträge inkl. } \\
\begin{array}{l}\text { Vorwörter und Einleitungen, } \\
\text { davon 9 ohne Beleg) }\end{array}\end{array}$ & 91 & 261 & 33 & 22 & 57 & 8 & 7 & 4 \\
\hline $\begin{array}{l}\text { Übersetzungen } \\
\text { (insges. 65 Beiträge, inkl. } \\
\begin{array}{l}\text { Vorwörter und Einleitungen, } \\
\text { davon 15 ohne Beleg) }\end{array}\end{array}$ & 30 & 157 & 12 & 5 & 12 & 2 & 1 & 3 \\
\hline
\end{tabular}

Tab. 1: Anzahl Belege in Originalen und Übersetzungen

\subsection{Kombinatorik: Im Zentrum die Klassiker und ihre Nachfolger}

Eines der Nebenprodukte der diversen Suchdurchgänge sind die Namen von Soziologen oder WissenschaftlerInnen benachbarter Disziplinen, die häufig zitiert werden. Im Kontext dieser Namensnennungen sind, so ist zu vermuten, neben den indirekten auch direkte Reden zu 
erwarten. Zu den Klassikern der Soziologie werden in den Annali Auguste Comte (17981857), Karl Marx (1818-1883), Vilfredo Pareto (1848-1923), Émile Durkheim (1858-1917), Ferdinand Tönnies (1855-1936), Georg Simmel (1858-1918), Max Weber (1864-1920) und Max Scheler (1874-1928) gezählt, während Talcott Parsons (1902-1979), Niklas Luhmann (1927-1998), Achille Ardigò (1921-2008), Michel Foucault (1926-1984) und Jürgen Habermas $(* 1929)$ als Nach-Klassiker bezeichnet werden. Daneben spielen die Philosophen Wilhelm Dilthey (1833-1911), Edmund Husserl (1859-1938) und Hans-Georg Gadamer (1900-2002) eine gewichtige Rolle.

\subsubsection{Name plus Hilfsverb bzw. Modalverb}

Diese Namen werden nun in Kombination mit den bereits untersuchten Formen der Hilfsbzw. Modalverben mittels Sketch engine gesucht. Es ist dies auch eine Art Kontrollsuche, um herauszufinden, in welche Kontexte die bereits gefundenen indirekten Reden gehören könnten. Da die Eigennamen vorläufig nur über die Funktion Phrase gesucht werden können, sind die Kombinationsmöglichkeiten der Anfragen relativ beschränkt. Die Hilfs- und Modalverben müssen als Lemma eingegeben werden, was, wie $\mathrm{zu}$ erwarten ist, zu vollkommen unspezifischen und unbefriedigenden Ergebnissen führt.

Tab. 2 gibt einen Überblick über die Ergebnisse dieser Suchfigur. Spalte 1 enthält die Anzahl der Belege für den ganzen Namen, d. h. mit Vor- und Nachnamen, Spalte 3 die Anzahl der Belege nur mit dem Nachnamen, die Spalten 4-11 beinhalten die Anzahl der belegten Hilfsbzw. Modalverben, und in Spalte 12 findet sich ein Hinweis auf das Vorhandensein direkter Reden (vgl. Tab. 2, Spalten 2, 3 und 12).

\begin{tabular}{|c|c|c|c|c|c|c|c|c|c|c|c|}
\hline 1 & 2 & 3 & 4 & 5 & 6 & 7 & 8 & 9 & 10 & 11 & 12 \\
\hline Anzahl $^{3}$ & $\begin{array}{r}\text { Belege } \\
\text { Vorname/ } \\
\text { Name }\end{array}$ & $\begin{array}{r}\text { Belege } \\
\text { nur } \\
\text { Name }\end{array}$ & thabe & + sei & +werde & +müsse & +könne & +dürfe & +solle & +wolle & $\begin{array}{r}\text { dir. } \\
\text { Rede }\end{array}$ \\
\hline $\begin{array}{l}\text { Auguste } \\
\text { Comte }\end{array}$ & 4 & 35 & 1 & 0 & 0 & 0 & 0 & 0 & 0 & 0 & 0 \\
\hline $\begin{array}{l}\text { Karl } \\
\text { Marx }\end{array}$ & 7 & 69 & 0 & (1) 1 & 0 & 0 & 0 & 0 & 0 & 0 & + \\
\hline $\begin{array}{l}\text { Vilfredo } \\
\text { Pareto }\end{array}$ & 5 & 15 & 0 & 1 & 0 & 0 & 0 & 0 & 0 & 0 & + \\
\hline $\begin{array}{l}\text { Émile } \\
\text { Durkheim }\end{array}$ & 4 & 37 & 0 & 0 & 0 & 0 & 0 & 0 & 0 & 0 & + \\
\hline $\begin{array}{l}\text { Ferdinand } \\
\text { Tönnies }\end{array}$ & 3 & 22 & 0 & 0 & 0 & 0 & 0 & 0 & 0 & 0 & + \\
\hline $\begin{array}{l}\text { Georg } \\
\text { Simmel }\end{array}$ & 30 & 603 & 3 & 1 & 2 & 0 & 0 & 0 & 0 & 0 & + \\
\hline $\begin{array}{l}\text { Max } \\
\text { Weber }\end{array}$ & 107 & 460 & (3)3 & (3) 1 & 0 & 0 & (5)1 & 0 & 0 & (1)0 & + \\
\hline $\begin{array}{l}\text { Max } \\
\text { Scheler }\end{array}$ & 6 & 60 & 0 & 0 & 0 & 0 & 0 & 0 & 0 & 0 & + \\
\hline $\begin{array}{l}\text { Talcott } \\
\text { Parsons }\end{array}$ & 16 & 11 & 0 & 0 & 0 & 0 & 0 & 0 & 0 & 0 & + \\
\hline $\begin{array}{l}\text { Niklas } \\
\text { Luhmann }\end{array}$ & 16 & 72 & 0 & 0 & 0 & 0 & 0 & 0 & 0 & 0 & + \\
\hline $\begin{array}{l}\text { Achille } \\
\text { Ardigò }\end{array}$ & 4 & 24 & 0 & 0 & 0 & 0 & 0 & 0 & 0 & 0 & + \\
\hline $\begin{array}{l}\text { Michel } \\
\text { Foucault }\end{array}$ & 0 & 15 & 0 & 0 & 0 & 0 & 0 & 0 & 0 & 0 & + \\
\hline $\begin{array}{l}\text { Jürgen } \\
\text { Habermas }\end{array}$ & 6 & 41 & 1 & 0 & 0 & 0 & 0 & 0 & 0 & 0 & + \\
\hline
\end{tabular}

\footnotetext{
3 Zahlen in Klammern entstammen der Suche Verbform = Phrase + Name im linken bzw. rechten Kontext.
} 


\begin{tabular}{|l|r|r|r|r|r|r|r|r|r|r|r|}
\hline $\begin{array}{l}\text { Wilhelm } \\
\text { Dilthey }\end{array}$ & 15 & 545 & 3 & 4 & 0 & 2 & 1 & 0 & 0 & 0 & + \\
\hline $\begin{array}{l}\text { Edmund } \\
\text { Husserl }\end{array}$ & 2 & 100 & 0 & 0 & 0 & 0 & 1 & 0 & 0 & 0 & + \\
\hline $\begin{array}{l}\text { Hans- } \\
\text { Georg } \\
\text { Gadamer }\end{array}$ & 0 & 38 & 0 & 0 & 0 & 0 & 0 & 0 & 0 & 0 & + \\
\hline
\end{tabular}

Tab. 2: Überblick über die Kombinationssuche

Was Tab. 2 auf den ersten Blick enthüllt: Diese kombinierte Suche hat nicht zu den gewünschten Resultaten geführt (Treffer in der Tabelle grau markiert). Der Aufwand ist groß und lohnt sich nicht wirklich. Was aber eine gezieltere Suche, bei der ein Eigenname auch als Lemma erkannt wird, leisten könnte, sollen im Folgenden die Bsp. Max Weber und Karl Marx zeigen.

\subsubsection{Desiderat: Tagging von Eigennamen}

Max Webers Name wie auch der von Karl Marx gehören offensichtlich zum lexikalischen Material, das durch den Tagger markiert wurde, warum auch immer. ${ }^{4}$ Kombiniert man "habe" als Phrase und "Weber" als Lemma in der Links- oder in der Rechtserweiterung mit dem Abstand von 15 Token, so wird man schnell fündig.

a) Phrase: "habe" plus "Weber" (Kontext links, Abstand 15 Token):



b) Phrase: "habe" plus "Weber" (Kontext rechts, Abstand 15 Token):
Home Concordance Word Sketch Thesaurus Sketch-Diff Frequency Collocation KWIC/Sentence View options Sample Filter Sort 琵琵琵

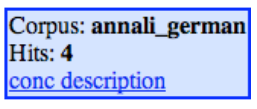

Hits: 4

1992b 05b habe, um eine Adoptivvaterschaft. Ich habe jedoch den Verdacht, daß sowohl Simmel

Wie die Ergebnisse zu a) und b) zeigen, finden sich insgesamt drei Belege für die indirekte Rede, in deren Nachbarschaft auch der Name Webers fällt. Um sicher zu gehen, wurden die gleichen Kombinationen auch als Name $=$ Phrase plus Hilfsverb bzw. Modalverb als Lemma eingegeben und die entsprechenden Belege handverlesen (vgl. Bsp. c) und d)). Wenn man die Ergebnisse in Bsp. a) und b) mit denen in Bsp. c) und d) vergleicht, kann man die Zeitersparnis ermessen, die die erstere, kombinierte und präzise Suche gegenüber allgemeineren Suchverfahren ermöglichen würde, falls sämtliche Eigennamen vom System als Lemma akzeptiert würden. Dass dies nicht der Fall ist, beweisen die zahlreichen variierten Kombinationsabfragen, die alle außer die mit "sei" plus "Marx" (rechter Kontext) nur ein Ergebnis liefern, nämlich "Empty result". So bleibt nur das aufwendige Suchen und Handverlesen der allzu freien Kombination Name $=$ Phrase plus Lemma des Verbs im linken oder rechten Kontext (vgl. Abb. 3), was umso enttäuschender ist, da das Beispiel Webers und Marx' zeigt, dass die Suchmaschine einfach zuverlässiger hinschaut als das menschliche Auge (vgl. Tab. 2, Zahlen in Klammern).

4 Es ist mir unergründlich, weshalb auch der Name "Marx" in diesem Zusammenhang funktioniert. Da die kombinierte Suche mit sämtlichen anderen Namen ergebnislos blieb, nahm ich an, dass der Name "Weber" vom System nicht als Name, sondern als Berufsbezeichnung interpretiert wird und deshalb Ergebnisse zu haben sind. 
Home Concordance Word Sketch Thesaurus Sketch-Diff

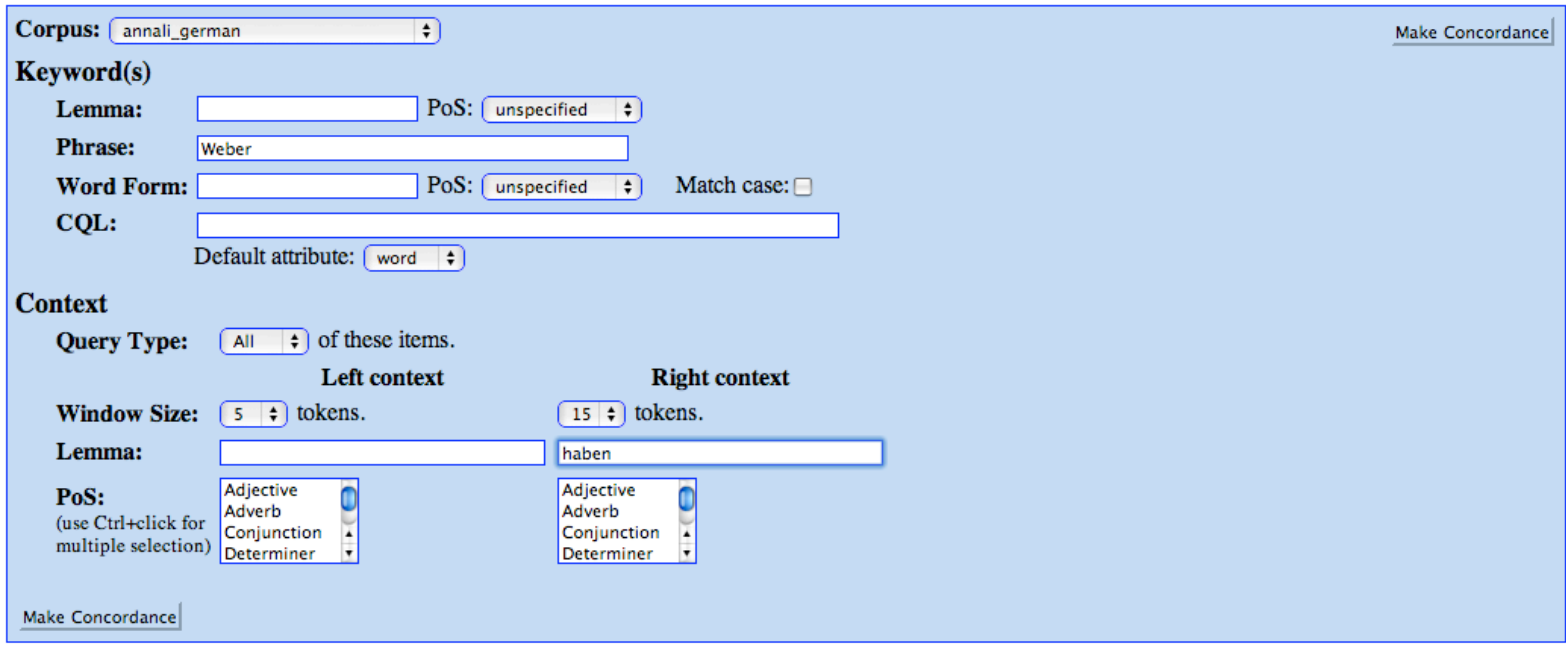

\section{Abb. 4: Suchmaske Sketch engine mit kombinierter Suche: Name plus Lemma des Verbs}

c) Phrase:"Weber" plus "haben" (Kontext links, Abstand 15 Token):

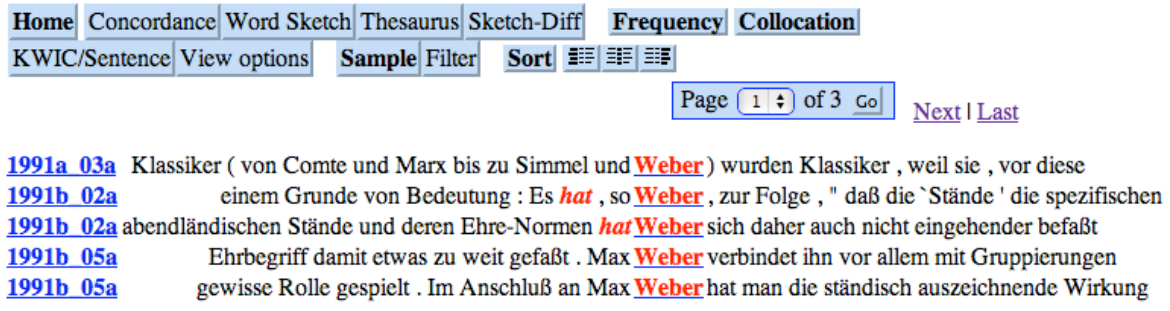

d) Phrase:"Weber" plus "haben" (Kontext rechts, Abstand 15 Token):

\section{Home Concordance Word Sketch Thesaurus Sketch-Diff Frequency Collocation KWIC/Sentence View options Sample Filter Sort 琵琵琵}

\section{Page $1 \div$ of 4 Go}

Corpus: annali_german
Hits: $\mathbf{4 7}$
conc description

\footnotetext{
1991a 14b Verbindung von Verstehen und Erklären bei Max Weber, mit der er uns eine fruchtbare und konstruktive 1991b 02a Autoren wie Tönnies, Simmel, Max und Alfred Weber hatten ihn als Bestandteil der sie umgebenden $1991 \mathrm{~b} 02 \mathrm{a}$ $1991 \mathrm{~b} 05 \mathrm{a}$ 1992a 01b 1992a 09a

1992a 09a 1992a 11a 1992a 11a

1992a 11a 1992a 11a $1992 \mathrm{~b} 04 \mathrm{a}$

1992b 05b 1992b 05b nur an Lukács und Bloch ) zu Simmel und Weber eingenommen hat . Die bereits zitierte 1992b 05b Methoden, und es ist bekannt, daß sogarWeber Simmel das Verdienst zuerkannte , eine 1992b 05b Aspekte scheinen mir jedoch beachtenswert. Weber und Simmel haben in der Pionierphase der 1992b 06a ähnlich denen, die er in seiner Kritik an Weber ins Feld geführt hatte : Eine solche Methode 1992b 09b Weiblichkeit. Weiterhin betont Marianne Weber, der Zugang zur objektiven Kultur habe 1993b 01a Weber-Literatur oft zu findenden Ansicht zu lesen, Weber habe mit seiner Verbindung von charismatischer

1993b 03b plebiszitären Demokratie " umreißen, die Weber nicht angemessen miteinander verbunden 1993b 03b Beispielen, die " den Typus geben ", erklären. Weber hat allerdings kontinuierlich Untersuchungen

1993b 03b ) Typus der Herrschaft, den dieses nach Weber ursprünglich hervorgebracht hat ( "die

1993b 03b " ( um einen Ausdruck aufzugreifen, den Weber einmal in Politik als Beruf verwendet hat 1993b 03b sicherstellen. Ich glaube sagen zu können, Weber habe dem Parlament hauptsächlich den Schutz
}

Die genaue Lektüre sämtlicher Belegstellen mit Eigennamen hat zudem ergeben, dass neben der indirekten Rede in den vorliegenden soziologischen Beiträgen andere Formen der Redewiedergabe häufiger verwendet werden. Die auf die Berühmtheiten des Faches Bezug nehmende Darlegung und Bewertung von Positionen wie auch die Wiedergabe und Einord- 
nung von deren Gedanken und Gedankengebäuden taucht vorwiegend in der im Indikativ Präsens formulierten Gestalt der Zusammenfassung auf und bedient sich, wenn die Rede des Anderen wiedergegeben werden soll, nur höchst selten der indirekten Rede, viel häufiger jedoch der direkten Rede, d. h. direkter Zitate. Insofern ist die Suche nach den Namen ein möglicher Weg, direkte Reden aufzufinden, insbesondere dann, wenn man den Namen mit einem Verbum dicendi koppelt, wie etwa "Dilthey" +"sagen".

e) Phrase: "Dilthey" + "sagen" (rechter Kontext, 5 Token):

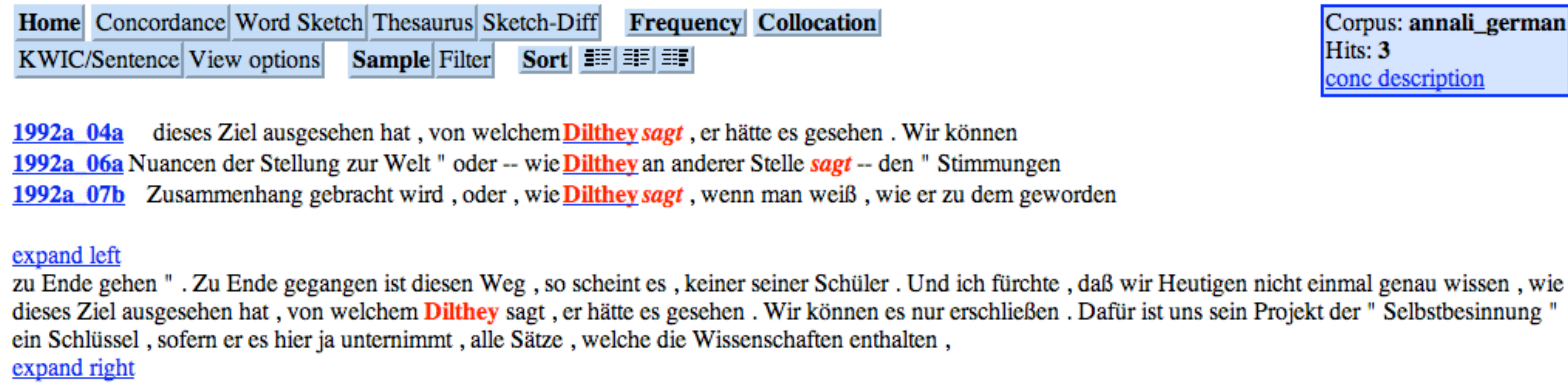

Verba dicendi leiten aber auch indirekte Reden ein, das belegt der Auszug aus 1992a 04a. Hier jedoch handelt es sich um einen Beleg für indirekte Rede, die mit den Formen des Konjunktiv II gebildet wurde. Sozialwissenschaftliche Experten des Schreibens halten sich offensichtlich nicht an die Regeln, die GrammatikerInnen aufgestellt haben, was die Ausgangshypothese, dass mit grammatikalischen Formen schnell zum Gesuchten vorzustoßen sei, relativiert. Eine neue Suchrunde, die Konjunktiv-II-Formen einbezieht, müsste nun also beginnen. Sie wäre aber angesichts der vielfältigen syntaktischen Funktionen des Konjunktivs II wiederum ein weites Feld für Handzuverlesendes.

\section{Suche und Ergebnis}

Dass am Ende dieser Suchfiguren neue Fragen stehen und neue Möglichkeiten des Suchens sich anbieten, bestätigt nur die Erkenntnis, dass die Antwort einer Maschine nicht klüger sein kann als die Frage, die an sie gerichtet wird. Wer durch korpuslinguistische Methoden Zugang zu textuellen Phänomenen sucht, ist darauf angewiesen, die Grenzen, die ihm oder ihr durch die vorgegebene bzw. gewählte Suchmaschine gesetzt sind, mit immer neuen kreativen Fragemethoden $\mathrm{zu}$ verschieben und zu überrumpeln und findet im glücklichen Falle, was er oder sie erwartet oder eben auch nicht gesucht hat.

Die hier durchgeführten Suchaktionen ergeben, in Kombination mit den Möglichkeiten von Excel, einen guten Überblick, wenn auch keineswegs vollständige Angaben, über umfangreichere Belegstellen für indirekte Rede im Korpus. Was der Gesamtüberblick (vgl. Diagramm 5) erkennen lässt, sind Häufungen in gewissen Beiträgen, aber nicht in einzelnen Bänden. Die Daten, die sich aus den durchgeführten Suchfiguren ergeben haben, lassen auf eine leichte Tendenz der Abnahme des Gebrauchs der indirekten Rede während der gut zehn Jahre, die das Korpus umfasst, schließen, einzelne Ausreißer mit höherer Beleganzahl gibt es aber auch in den späteren Bänden. Was die zahlenmäßig unterschiedliche Belegverteilung zwischen den deutschsprachigen Originalen und den Übersetzungen aus dem Italienischen vermuten lässt, sind gewisse Unterschiede im Gebrauch der Redewiedergabe in den beiden Sprach- und Wissenschaftstraditionen. Die hier erhaltenen Ergebnisse sind aber zu ungenau, um seriöse Hypothesen zu erlauben. Es bedarf dazu genauerer Einzeluntersuchungen an den Originalen und den entsprechenden Übersetzungen. 


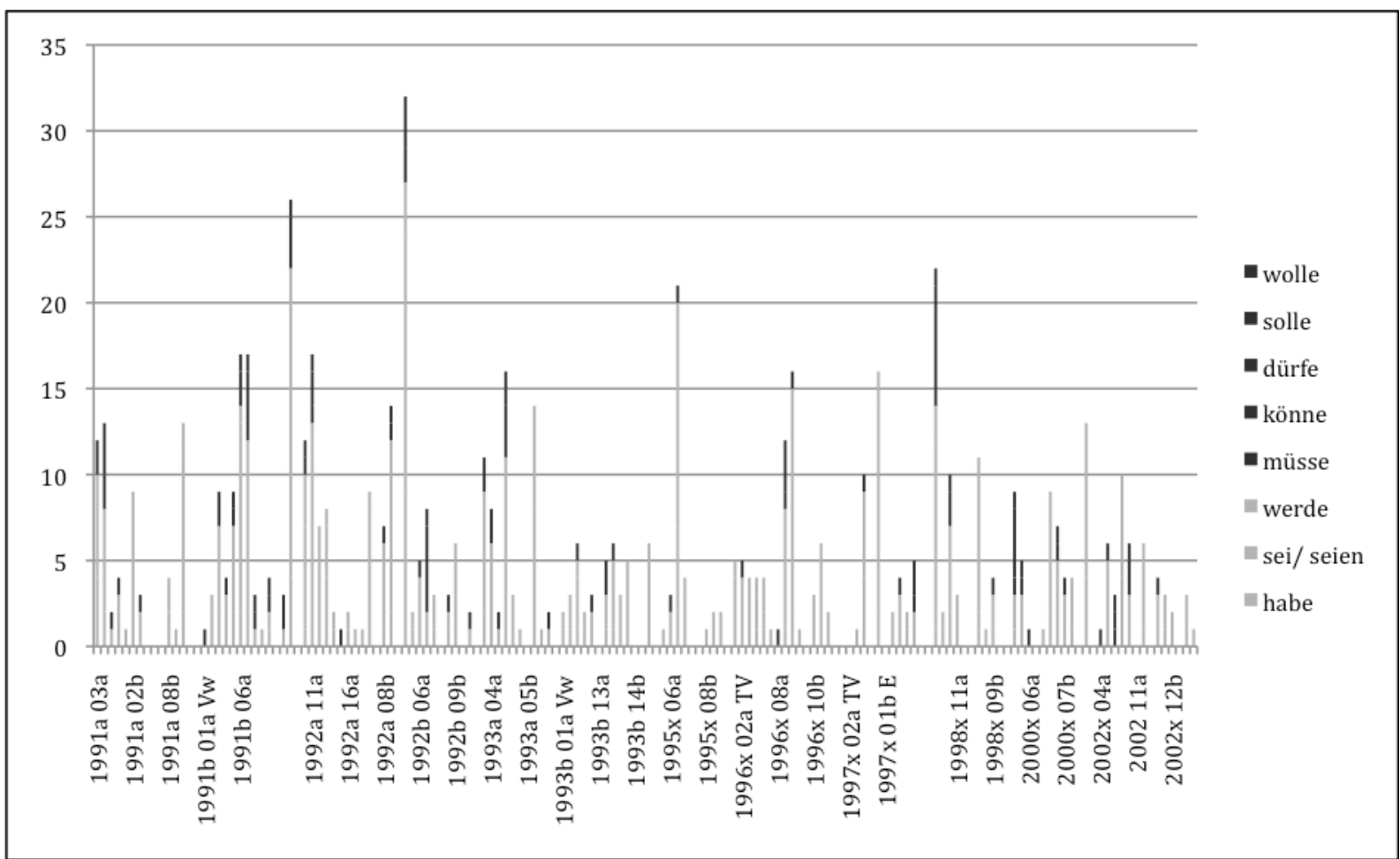

Diagramm 5: Gesamtüberblick über die Verteilung der Belege pro Beitrag und Band (Hilfsverben gesamt: hellgrau: Modalverben gesamt: dunkelgrau)

Nach Abschluss dieser Arbeit bieten sich diverse weitere Suchfiguren an, die das Gefundene vermutlich ergänzen werden. Die sicherlich nötige Suche nach Verba dicendi plus indirekter Rede verspricht neue Akzente, aber auch einiges an "philologischer Handarbeit" (vgl. Näf 2006: 106), die, wie sich während des Arbeitens an den unterschiedlichen, hier dargestellten Suchfiguren gezeigt hat, zu einem merkwürdigen "Zappeltext" (Schmitz 2000) im Gehirn der forschenden Person führen. Die Fixierung der lesenden Augen auf das Gesuchte, das auf der Suchmaske in einer Fülle verschiedenster Fragmente erscheint, verschafft dem biologischen Speicher einen Zettelkasten von Einzelinformationen, die ein Kohärenz suchender Geist immer wieder aufeinander zu beziehen scheint. Was hierbei entsteht, ist in der Tat ein "Zappeltext" im Sinne Schmitz'; im vorliegenden Fall ist es ein Konglomerat von indirekten Aussagen, ein Konglomerat der korpuslinguistisch organisierten Vereinzelung dessen, was in all den Originalen ja einmal in komplexem Zusammenhang dargelegt wurde. Was also als soziologischer Fachtext wahrgenommen wird, was sich implizit als Textmustervorstellung konstruieren könnte, entfernt sich aufgrund der Methodik notwendigerweise von dem, was der Fachtext in Wirklichkeit ist. Damit scheint sich die Erkenntnis Bahn zu brechen, dass die korpuslinguistische Methode für die Untersuchung von textuellen Phänomenen lediglich Hilfsinstrument, nicht aber federführend sein kann, vor allem dann nicht, wenn die technischen Möglichkeiten noch zu wenig ausgereift sind, um präzise, zuverlässige und Zeit sparende Abfragen zu ermöglichen.

\section{Literaturangaben}

Adamzik, Kirsten (1998): "Intertextualität in der wissenschaftlichen Kommunikation". In: Strässler, Jürg (ed.): Tendenzen europäischer Linguistik. Akten des 31. Linguistischen Kolloquiums Bern 1996. Tübingen: 1-5. (= Linguistische Arbeiten 381).

Associazione Italo-Tedesca di Sociologia/Italienisch-Deutsche Gesellschaft für Soziologie (ed.) (1985-): Annali di Sociologia/Soziologisches Jahrbuch. Berlin etc. 
Bachtin, Michail M. (1979): Die Ästhetik des Wortes. Übersetzung aus dem Russischen von Rainer Grübel und Sabine Reese; hrsg. von Rainer Grübel. Frankfurt am Main.

Heller, Dorothee (ed.) (2008a): Formulierungsmuster in deutscher und italienischer Fachkommunikation. Intra- und interlinguale Perspektiven. Bern etc. (= Linguistic Insights 89).

Heller, Dorothee (2008b): "Kommentieren und Orientieren. Anadeixis und Katadeixis in soziologischen Fachaufsätzen". In: Heller, Dorothee (ed.): Formulierungsmuster in deutscher und italienischer Fachkommunikation. Intra- und interlinguale Perspektiven. Bern etc.: $105-138$.

Jakobs, Eva-Maria (1994): "Conceptsymbols. Funktionen von Zitation und Verweisung im wissenschaftlichen Diskurs". In: Halwachs, Dieter W./Stütz, Irmgard (eds.): Sprache Sprechen - Handeln. Akten des 28. Linguistischen Kolloquiums Graz 1993. Bd. 2. Tübingen: $45-52$.

Näf, Anton (2006): "Satzarten unterscheiden - Kann das der Computer? Syntaktische Explorationen anhand von COSMAS II". Linguistik online 28/3: 85-107.

Panther, Uwe (1981): "Indirekte sprachliche Handlungen im wissenschaftlichen Diskurs". In: Bungarten, Theo (ed.): Wissenschaftssprache. München: 231-260.

Pérennec, Marie-Hélène (2000/2003): "Das Konzept der Polyphonie als Instrument der Textinterpretation". LYLIA 2. langues.univ-lyon2.fr/sites/langues/IMG/pdf/doc-190.pdf (Stand: 01.03.2009).

Schmitz, Ulrich (2000): "ZAP und Sinn. Fragmentarische Textkonstitution durch überfordernde Medienrezeption". In: Ernest W. B. Hess-Lüttich et al. (eds.): Textstrukturen im Medienwandel. Frankfurt am Main etc.: 11-29.

Zanin, Renata (2007a): "Parlare come gli altri". Il Cristallo 49/1: 100-104.

Zanin, Renata (2007b): "Korpusinstrumente für Deutsch als Zweitsprache". In: Krumm HansJürgen/Portmann-Tselikas Paul R. (eds.): Theorie und Praxis. Österreichische Beiträge zu Deutsch als Fremdsprache 10. Innsbruck: 83-100.

Zifonun, Gisela/Hoffmann, Ludger/Strecker, Bruno et al. (1997): Grammatik der deutschen Sprache. 3 Bde. Berlin/New York. 